\title{
Multi-Drug and Pan-Drug Resistant Pseudomonas aeruginosa: A Challenge in Post- Antibiotic Era
}

\author{
Shiva Bhandari ${ }^{1}$, Megha R. Banjara ${ }^{1}$, Binod Lekhak ${ }^{1}$, \\ Dwij R. Bhatta ${ }^{1}$ and \\ Shyam R. Regmi \\ ${ }^{1}$ Central Department of Microbiology \\ Tribhuvan University, Kirtipur, Kathmandu \\ ${ }^{2}$ Shahid Gangalal National Heart Center \\ Bansbari, Kathmandu \\ e-mail: vigourshiva@hotmial.com
}

\begin{abstract}
Clinicians still lack an appreciation of the potential importance of Pseudomonas aeruginosa (PA) in the hospitals. So this study was done in order to isolate and assess the prevalence of multi-drug resistant $P$. aeruginosa (MDRPA) and pan-drug resistant $P$. aeruginosa (PDRPA) from the intensive care units (ICUs). A cross-sectional study was conducted for six months at the ICUs of Shahid Gangalal National Heart Centre, Kathmandu. A total of 1,060 samples were processed out of which 700 were clinical samples directly taken from the admitted patients while 360 were the surface swab samples. The isolates were identified using routine conventional methods based upon microscopic findings, colony morphology and biochemical properties. Antibiotic susceptibility testing (AST) was performed by Kirby Bauer disc diffusion technique. P. aeruginosa was isolated from 66 (9.43\%) clinical samples while 60 (16.67\%) were surface swab samples. Among clinical isolates, 56 (84.8\%) were sensitive to cefoparazone+sublactam (CSL) followed by 42 (63.6\%) to polymixin-B and 36 (54.5\%) to piperacillin-tazobactam (PT), while among surface swab sample isolates more than $90 \%$ were sensitive to most of the common antibiotics used. About 59 (89.4\%) MDRPA were isolated from clinical samples while it was only 7 (11.7\%) from surface swab samples. Only isolates from clinical samples (6.1\%) yielded PDRPA. Since $P$. aeruginosa has shown the reduced susceptibility towards single antibiotics such as carbapenems, fluoroquinolones, aminoglycosides and cephalosporins, combined antibiotics like CSL and PT remained choice of treatment. This can eliminate potential threat of MDRPA and PDRPA in the ICUs.
\end{abstract}

Key words: multi-drug resistance, pan-drug resistance, Pseudomonas aeruginosa

\section{Introduction}

The emergence of antibiotic resistance in Pseudomonas aeruginosa (PA) and its spread has been a serious challenge to the clinicians. The bacterium is the fourth most commonly-isolated nosocomial pathogen accounting for $10.1 \%$ of all hospital-acquired infections (Todar 2011). The risk of nosocomial infection in ICU is 5-10 times greater than those acquired in general medical and surgical wards (Vincet et al. 1995). Nosocomial infections caused by $P$. aeruginosa are very common; $11-13.8 \%$ of all nosocomial infections are due to $P$. aeruginosa, with an even higher rate (13.2-22.6\%) of nosocomial infections among patients in an intensive care unit
(ICU) (Driscoll et al. 2007). Since $P$. aeruginosa is one of the most common flora of the ICUs and due to its long persistence (on dry inanimate surfaces: 6 hours 16 months; on dry floor: 5 weeks) (Kramer et al. 2006). The chances of infection by this organism increase rapidly. In addition, the recurrent patient acquisition of $P$. aeruginosa has been linked to wide-spread environmental contamination by this bacterium (Fanci et al. 2006).

P. aeruginosa is a clinically significant pathogen, often causing nosocomial infections in immunocompromised and catheterized patients. In fact, $P$. aeruginosa is the epitome of an opportunistic 
pathogen of humans. It generally produces bluishgreen and yellowish-green colonies, which produce typical grape-like odor because of 2aminoacetophenone (Moore \& Hancock 2011).

Although in the beginning strains of $P$. aeruginosa are sensitive to various classes of antibiotics, several studies have documented increasing resistance rates to fluoroquinolones, cephalosporins and carbapenems, particularly among ICU isolates (CDC 2004, Streit et al. 2004, Jones et al. 2004). The overall prevalence of antibiotic resistant $P$. aeruginosa is increasing, with up to $10 \%$ of global isolates found to be multidrug-resistant (Gales et al. 2001). Likewise in a study done in India the prevalence of PDRPA was up to 4\% (Jayakumar \& Appalaraju 2007). Prevalence of MDRPA, PDRPA and their antibiotic profile was investigated to select an appropriate empirical therapy. Moreover, lack of sufficient data on prevalence of MDRPA and PDRPA in tertiary care hospitals indicated the need for this study.

\section{Methodology}

A total of 1,060 samples were processed during a six month study period from November, 2010 to May, 2011 in ICUs at Shahid Gangalal National Heart Centre, Kathmandu a tertiary care heart hospital. Among 700 clinical samples, there were 160 ET tube tips, 142 suction tube tips, 151 Foley's tube tips, 160 CVP tube tips, 30 sputum, 20 wound swabs, 8 pus, 25 blood and 4 tracheal secretion samples. Among 360 surface swab samples, 90 from each floor, bed lining, working tables and aprons of the HCWs were taken in the study.

The clinical samples were collected in a sterile, leak proof and well capped container with the help of medical staff working in the wards. As soon as the samples were collected they were transported to the laboratory immediately and microbiological processing was carried out.

Swabs from the floor, bed linings, working table and apron of the HCWs, who were working in the ICUs were taken. Sterilized cotton plugged swabs dipped into normal saline were used to collect sample for investigation from inanimate objects. Using a sterile cotton swab dipped in normal saline, the surfaces were swabbed revolving the swab in ' $S$ ' shaped fashion so as to collect the remains of microbial load (Sehulster 2008). For the purpose of quality control each batch of swab was tested for sterility by streaking on culture medium and incubation at $37^{\circ} \mathrm{C}$ for 24 hours to observe colonies if growth occurred. For proper collection of samples it was made sure that the swab was revolved during the procedure. All the samples were collected aseptically.

The isolates were identified using routine conventional methods based upon microscopic findings, colony morphology and biochemical properties. Routine antibiotic sensitivity testing was done by Kirby Bauer disc-diffusion method according to the recommendations of Clinical and Laboratory Standard Institute (CLSI 2011).

\section{Results and Discussion}

A total of 1,060 samples from the ICUs were processed. Among 700 clinical samples, 66 yielded $P$. aeruginosa. Likewise, among 360 surface swab samples, 60 yielded $P$. aeruginosa. Thus the prevalence of $P$. aeruginosa was $9.43 \%$ in the clinical samples while in case of surface swab samples it was $16.67 \%$.

Most isolates of $P$. aeruginosa isolated from clinical samples were highly resistant towards CFM, 65(98.5\%) followed by CTR and CAZ, both 62 (93.9\%). However, the isolates were sensitive towards CSL, 56 (84.8\%) followed by PT, 36 (54.5\%) and MEM, 34 (51.5\%). Most isolates of $P$. aeruginosa isolated from the surface swab samples were highly sensitive towards CFM, CAZ and POL, all 60 (100.0\%) effective. Very less isolates were resistant towards the antibiotics (Fig. 1).

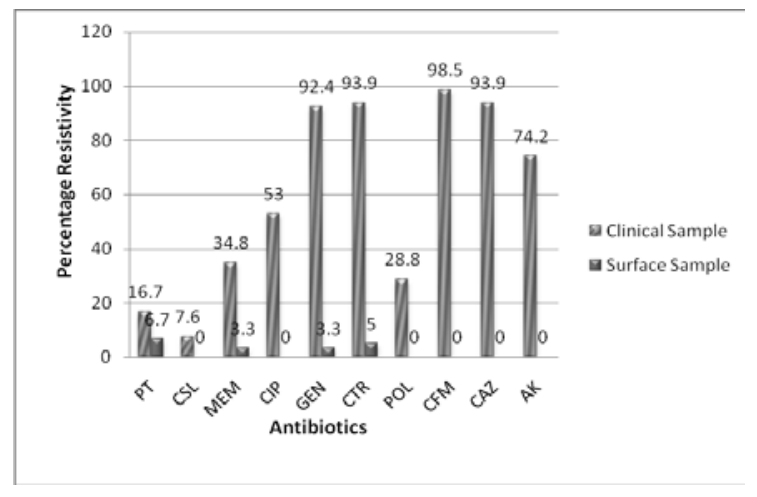

Fig.1. Resistivity pattern from clinical and surface samples (Note: PT= Piperacillin-tazobactam, CSL= cefoparazonesulbactam, $\mathrm{MEM}=$ meropenem, $\mathrm{CIP}=$ ciprofloxacin, $\mathrm{GEN}=$ gentamicin, $\mathrm{CTR}=$ ceftriaxone, $\mathrm{POL}=$ polymixin $-\mathrm{B}, \mathrm{CFM}=$ cefixime, $\mathrm{CAZ}=$ ceftazidime, $\mathrm{AK}=$ amikacin) 
When we studied the MDR pattern of the organism, we got comparable results. Fifty-nine (89.4\%) were MDRPA isolates from the clinical samples while only 7 (11.7\%) were MDRPA isolates from surface samples. No PDRPA isolates were obtained in case of surface samples while 4 (6.1\%) isolates were PDRPA among the clinical samples (Fig. 2).

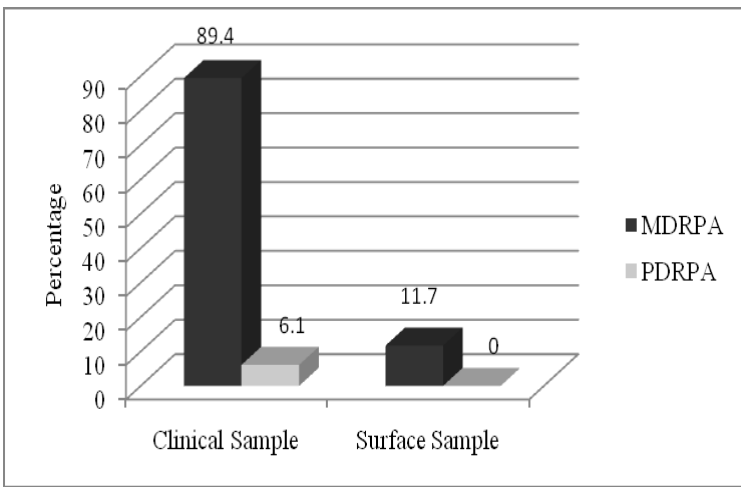

Fig. 2. MDRPA and PDRPA isolates

Among the various classes of antibiotics used, $P$. aeruginosa against cephalosporins registered highest resistance rate (100.0\%) followed by aminoglycosides (95.5\%) in case of isolation from clinical samples. We obtained similar results in case of surface swab samples as well; $P$. aeruginosa resistant to cephalosporins (25.0\%) and aminoglycosides (11.7\%) (Fig. 3).

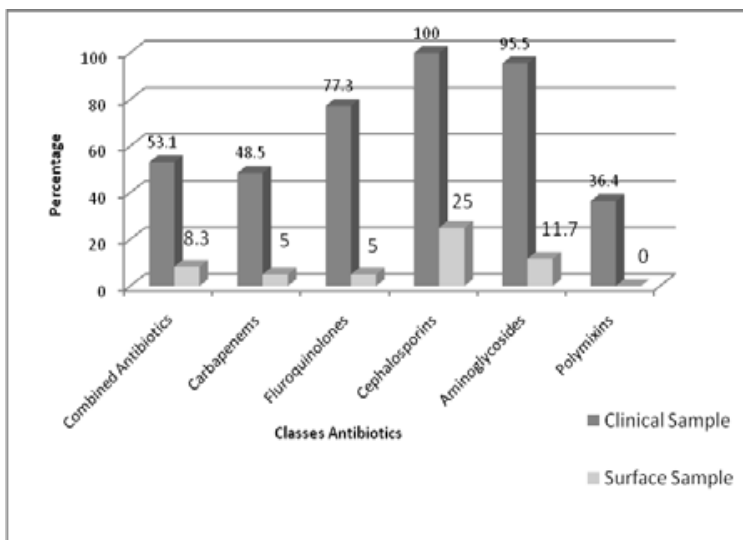

Fig. 3. Resistivity pattern of $P$. aeruginosa against various classes of antibiotics

$P$. aeruginosa is one of the most common pathogens that have posed threat to the ICU patients in the hospital. The environment in which patients were kept harbored $P$. aeruginosa. In our study, the aprons of HCWs, working tables, floor, all harbored $P$. aeruginosa, the sources of which can be exogenous or endogenous. Different studies (Karki et al. 2011, Sharma 2006) revealed that the organism as an opportunistic environment pathogen. In addition, the persistence of $P$. aeruginosa in the inanimate surfaces of the hospital environment increases the chances of infections. (Kraner et al. 2006)

Occurrence of $P$. aeruginosa in indwelling catheters has significant role in the pathogenesis of the organism especially in ICU patients. Due to the production of alginate slime the organism attaches itself to the surfaces of the catheters so that simple mechanical forces cannot remove them from the surfaces. The prevalence of mucoid strains in ICU patients has also been mentioned by various workers (Doggett 1969, Hoiby 1975). So, there are high chances of pseudomonal infections with the use of indwelling catheters. Therefore, catheters should be used under highly aseptic conditions and changed at proper intervals.

What we observed was the organism isolated from surface swabs became sensitive to almost all of the antibiotics used. POL, CFM and CAZ showed cent percent effectiveness while rest of others showed more than $90 \%$ effectiveness except CTR, which was $75 \%$ effective. In its natural habitat, $P$. aeruginosa did not receive any type of antibiotics. So the organism had not undergone changes in any of the systems to combat the effects of the drugs. In their natural habitats, most of them did not produce slime layer of alginate polysaccharide (non-mucoid) and it leads to the reduction of the biofilm production rendering vulnerable to antibiotics. There is increased resistance of organism due to biofilm production (Stewart \& Costerton 2001).

$P$. aeruginosa, with the profound use of various antibiotics, had changed itself into a stubborn organism, resistant to almost all the antibiotics. It showed resistance to CFM (98.5\%), CTR and CAZ (93.9\%), GEN (92.4\%), MEM (34.8\%), CIP (53.0\%) and POL (28.8\%). Resistance was observed even in case of combined antibiotics, PT (16.7\%) and CSL (7.6\%). A ten year study also suggests about the increasing pattern of the antibiotic resistance to various drugs against $P$. aeruginosa (Obritsch et al. 2004). In the MYSTIC 2006 results, among 1,012 P. aeruginosa isolates collected from 40 European centers, resistance 
to PT was the lowest (15\%), followed by meropenem (22\%), amikacin (23\%), ceftazimide (25\%), gentamicin (29\%) and ciprofloxacin (33\%) (Turner 2008).Thus, the organism is sensitive towards combined antibiotics to some extent while single antibiotics are being futile in the treatment of pseudomonal infections. Thus emphasis should be given towards combined antibiotics in the treatment of pseudomonal infections.

Multi-drug resistant $P$. aeruginosa (MDRPA) strains are the most challenging organisms for the clinicians to be overcome. In our study $89.4 \%$ of the total isolates from the clinical samples were MDRPA which is similar to a study carried out in Nepal (Koirala et al. 2010. In a recent study carried out in a tertiary care hospital in Nepal (Karki et al. 2011), MDRPA were also isolated from the clinical samples. This indicates the alarming situation of MDR pseudomonal infections in ICUs of various parts of Nepal. Out of 66 isolates 6.1\% were PDRPA isolates. This sort of complete (or almost complete) lack of treatment options is an increasingly common and desperate occurrence in tertiary care medical centers. The resistance pattern is increasing in such a way that evolution of Pan Drug Resistance $P$. aeruginosa occurred (Hsueh et al. 1998, Wang et al. 2006). So, rational use of antibiotics should be practiced.

Pseudomonal infection is still one of the most common nosocomial infections in the ICUs of Nepal, where floors can serve as the important source of infections. P. aeruginosa has shown the reduced susceptibility towards carbapenems, fluoroquinolones, aminoglycosides and cephalosporins. Since polymixins are toxic to human cells, combined antibiotics like CSL and PT remain the choice of treatment. Further, $P$. aeruginosa possesses more tools for defying the activity of antimicrobial agents than virtually any other microorganism, due to which no adequate therapeutic options exist; it is time to intensify attention towards the threats posed by pseudomonal resistance.

\section{Acknowledgements}

We greatly acknowledge the support provided by Shahid Gangalal National Heart Center and the
Central Department of Microbiology, Tribhuvan University.

\section{References}

Centers for Disease Control and Prevention. 2004. National nosocomial infections surveillance (NNIS) system report, data summary from January 1992 through June 2004, issued October 2004. Am. J. Infect. Cont. 32: 470-485.

Clinical and Laboratory Standards Institute. 2011. Performance Standards for Antimicrobial Disk Susceptibility Testing; Twentieth Informal Supplement M100-S21. 31 (1), CLSI, Villanova, PA.

Doggett, R.G. 1969. Incidence of mucoid Pseudomonas aeruginosa from clinical sources. Appl. Environ. Microbiol. 18 (5): 936-937.

Driscoll, J.A., S.L. Brody and M.H. Kollef. 2007. The epidemiology, pathogenesis and treatment of Pseudomonas aeruginosa infections. Drugs 67: 351368.

Fanci, R., P. Pecile, E. Casalone et al. 2006. Pseudomonas aeruginosa sepsis in stem cell transplantation patients. Infect. Control. Hosp Epidemiol. 27: 767770.

Gales, A.C., R.N. Jones, J. Turnidge, R. Rennie and R. Ramphal. 2001. Characterization of Pseudomonas aeruginosa isolates: occurrence rates, antimicrobial susceptibility patterns, and molecular typing in the global SENTRY Antimicrobial Surveillance Program, 1997-1999. Clin. Infect. Dis. 32: 146-155.

Hoiby, N. 1975. Prevalence of mucoid strains of Pseudomonas aeruginosa in bacteriological specimens from patients with cystic fibrosis and patients with other diseases. Acta. Pathol .Microbiol. Scand. Suppl. 83(6): 549-552.

Hsueh, P.R., L.J. Teng, P.C. Yang et al. 1998. Persistence of a multidrug-resistant Pseudomonas aeruginosa clone in an intensive care burn unit. J. Clin. Microbiol. 36: 1347-1351.

Jayakumar, S. and B. Appalaraju. 2007. Prevalence of multi and pan drug resistant Pseudomonas aeruginosa with respect to ESBL and MBL in a tertiary care hospital. Indian J. Pathol. Microbiol. 50(4): 922-925.

Jones, M.E., D.C. Draghi, C. Thornsberry et al. 2004. Emerging resistance among bacterial pathogens in the intensive care unit-a European and North American surveillance study (2000-2002). Ann. Clin. Microbiol. Antimicrob. 3: 14.

Karki, M., D.R. Bhatta, B.M. Pokhrel and U. Devkota. 2011. Prevalence of multi drug resistant Pseudomonas aeruginosa in the intensive care unit (ICU) patients and its association with indoor environment in tertiary care hospital in Nepal. M.Sc. dissertation. Tribhuvan University, Kirtipur. 
Koirala, P., D.R. Bhatta, P. Ghimire, B.M. Pokhrel and U. Devkota. 2010. Bacteriological profile of tracheal aspirates of the patients attending a neuro-hospital of Nepal. Int. J. Life. Sci. 4: 60-65.

Kramer, A., I. Schwebke and G. Kampf. 2006. How long do nosocomial pathogens persist on inanimate surfaces? A systematic review. Infect. Dis. 6: 130.

Moore, R.A. and R.E. Hancock. 1986. Involvement of outer membrane of Pseudomonas cepacia in aminoglycoside and polymyxin resistance. Antimicrob. Agents. Chemother. 30: 923-926.

Obritsch, M.D., D.N. Fish, R. MacLaren and R. Jung. 2004. National surveillance of antimicrobial resistance in Pseudomonas aeruginosa isolates obtained from intensive care unit patients from 1993 to 2002. Antimicrob. Agents. Chemother. 48(12): 4606-4610.

Sehulster, L. 2008. Environmental sampling in healthcare settings: A webber training teleclass. Center for Disease Control and Prevention.

Sharma, S. 2006. Microbial flora among visitors and the hospital environment in ICU and SICU at TUTH. M.Sc. dissertation. Tribhuvan University, Kirtipur.

Stewart, P.S. and J.W. Costerton. 2001. Antibiotic resistance of bacteria in biofilms. Lancet 358: 135-138.

Streit, J.M., R.N. Jones, H.S. Sader et al. 2004. Assessment of pathogen occurrences and resistance profiles among infected patients in the intensive care unit: report from the SENTRY antimicrobial surveillance program. Int. J. Antimicrob. Agents. 24: 111-118.

Todar, K. 2011. Todar's online book of bacteriology: 1-4 http://textbookofbacteriology.net/pseudomonas.html

Toutain, C.M., M.E. Zegans and G.A. O’Toole. 2005. Evidence for two flagellar stators and their role in the motility of Pseudomonas aeruginosa. J. Bacteriol. 187: 771-777.

Turner, P.J. 2008. Meropenem activity against European isolates: Report on the MYSTIC (Meropenem Yearly Susceptibility Test Information Collection) 2006 results. Diagn. Microbiol. Infect. Dis. 60(2): 185-192.

Victoria, L.C., J.L. Nicolás, G. Martha, D. Gerd, M.L. Tomás and B.P. Gerald. 2010. Evaluation of flagella and flagellin of Pseudomonas aeruginosa as vaccines. Infect. Immun. 78(2): 746-755

Vincent, J.L., D.J. Bihari, P.M. Suter write full authors et al. 1995. The prevalence of nosocomial infection in intensive care units in Europe. Results of the European prevalence of infection in intensive care (EPIC) study. EPIC International Advisory Committee. JAMA 274: 639-644.

Wang, C.Y., J.S. Jerng, K.Y. Cheng et al. 2006. Pandrugresistant Pseudomonas aeruginosa among hospitalised patients: clinical features, risk-factors and outcomes. Clin. Microbiol. Infect. 12(1): 63-68. 
Nepal Journal of Science and Technology Vol. 13, No. 2 (2012) 197-202 